EPJ Web of Conferences 61, 04011 (2013)

DOI: $10.1051 /$ epjconf/ 20136104011

(C) Owned by the authors, published by EDP Sciences, 2013

\title{
Study of High Energy Emission from Relativistic Jets with VERITAS
}

\author{
R. Mukherjee ${ }^{1, a}$ for the VERITAS Collaboration \\ ${ }^{1}$ Department of Physics and Astronomy, Barnard College, Columbia University, NY 10027, USA
}

\begin{abstract}
VERITAS is an imaging atmospheric Cherenkov telescope array carrying out observations of the gamma-ray sky at energies above $100 \mathrm{GeV}$. Located in southern Arizona, VERITAS consists of an array of four telescopes, and carries out a comprehensive observing program for Galactic and extragalactic high energy sources. Observations of astrophysical objects in the $\mathrm{TeV}$ band are sensitive probes of highly energetic processes occurring in these sources. The majority of the extragalactic sources detected at $\mathrm{TeV}$ energies are active galaxies of the blazar class, sources where we view the jet nearly along its axis. In this report we present recent results from the VERITAS extragalactic program, focusing on blazars.
\end{abstract}

\section{Introduction: VERITAS}

VERITAS (Very Energetic Radiation Imaging Telescope Array System) is an imaging atmospheric Cherenkov telescope array: it detects Cherenkov radiation from energetic particles, produced by the interaction of a gammaray primary in the atmosphere, using large optical reflectors on the ground. VERITAS operates at the base camp of the Fred Lawrence Whipple Observatory (FLWO) in southern Arizona. There are four 12m-diameter telescopes of Davies-Cotton design in the VERITAS array, with a camera comprising 499 photomultiplier tubes (PMTs) arranged in a hexagonal pattern, at the focal plane of each telescope. The total field of view has a diameter of $3.5^{\circ}$. Gamma-rays interact in the Earth's atmosphere before reaching the ground, producing electromagnetic cascades of relativistic electron-positron pairs which generate short-lived but bright flashes of Cherenkov radiation. Cosmic rays, the principal source of background, also produce showers of charged particles and Cherenkov radiation but at a rate much higher than that of gammaray showers. Imaging Atmospheric Cherenkov Telescopes (IACTs) such as VERITAS utilize the Cherenkov light emitted from these air showers to form an image of the longitudinal development of the air shower on the cameraplane. The use of the four VERITAS telescopes in combination helps to increase the collection area and obtain a stereoscopic image of the particle showers to reduce cosmic ray background. VERITAS has a sensitive energy range from $85 \mathrm{GeV}$ to $30 \mathrm{TeV}$ and an angular resolution of $<0^{\circ} .1$ at $1 \mathrm{TeV}$. Spectral reconstruction begins at $\sim 150$ $\mathrm{GeV}$ with an energy resolution $\sim 15 \%-20 \%$. Figure 1 is a photograph of the VERITAS observatory, showing the array of four telescopes. Details of the VERITAS telescopes are provided elsewhere [1]. Observations performed with VERITAS have led and will continue to lead to significant

\footnotetext{
ae-mail: rmukherj@barnard.edu
}

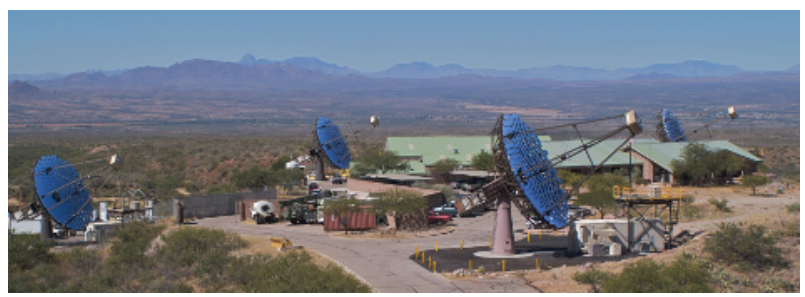

Figure 1. The four VERITAS telescopes located at the base camp of the Whipple Observatory in southern Arizona.

progress in our understanding of the accelerators of very high energy particles in the universe.

VERITAS started four-telescope operations in 2007, and typically collects more than $\sim 1100$ hours of data per year (including $\sim 200$ hours data taken in moderate moonlight). A $30 \%$ improvement in sensitivity was achieved in 2009 when one of the telescopes was relocated to provide a more symmetric array layout, and a new alignment system was used which improved the instrument point spread function [2]. In this new telescope arrangement, VERITAS is able to detect a source with a Crab Nebula-like spectrum and a flux of $1 \%$ Crab Nebula strength in about 25 hours. In the summer of 2012 the VERITAS cameras were replaced with new high-quantum efficiency (hQE, $\mathrm{QE}>32 \%$ ) photomultiplier tubes [3] that increased the photon detection efficiency of each camera by approximately $50 \%$. This is part of a series of upgrades and improvements that have been implemented on the VERITAS observatory since 2007 (see [4] for details on the gammaray detection sensitivity of the upgraded VERITAS observatory). Figure 2 is a photograph of one of the VERITAS cameras showing the new hQE PMTs.

VERITAS is normally operated using the full fourtelescope array. A multi-level trigger system is employed 


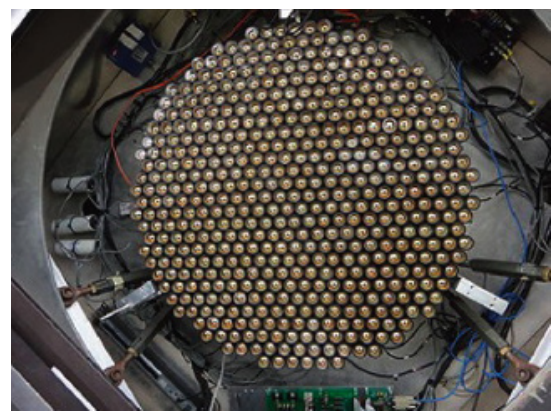

Figure 2. Photograph of one of the VERITAS cameras showing the new hQE PMTs (July 2012).

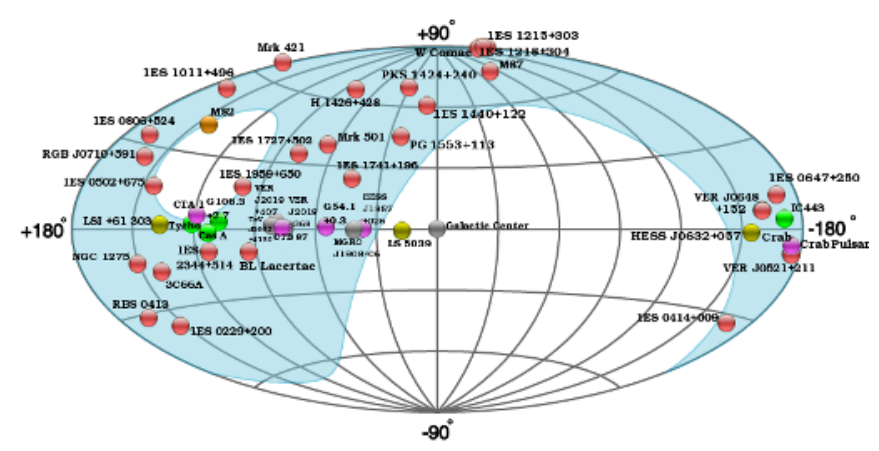

Figure 3. The VERITAS sky map of detected sources as of May 2013, in Galactic coordinates. The shaded area shows the region of visibility for VERITAS above 50 degree elevation. The majority of the detected sources off the Galactic plane are blazars (shown in red).

to eliminate background noise - at the array-level, a trigger requires simultaneous Cherenkov images in at least two telescopes, within a $50 \mathrm{~ns}$ time window, which then causes a readout of the $500 \mathrm{MSample/s} \mathrm{Flash-ADC} \mathrm{data}$ acquisition system for each pixel [5]. To date VERITAS has detected 46 Galactic and extragalactic sources, including blazars, pulsar wind nebulae (PWNe), supernova remnants (SNR), binary systems, a starburst galaxy and the Crab Pulsar. Figure 3 shows all sources detected by VERITAS, in a galactic projection. In this article, we review some of the results from VERITAS focusing on blazars and extragalactic sources.

\section{Extragalactic TeV Gamma-ray Sources detected by VERITAS}

The majority of the extragalactic sources detected by VERITAS are active galaxies of the blazar class. Active galactic nuclei (AGN) are cores of galaxies believed to contain supermassive black holes (SMBHs) accreting matter and generating non-thermal emission which often outshines the host galaxy. Relativistic jets are extremely powerful outflows of collimated plasma that appear in active galactic nuclei. In addition to AGN, relativistic jets also

\begin{tabular}{|c|c|c|}
\hline $\begin{array}{l}\text { Source Name } \\
\text { (Extragalactic) }\end{array}$ & Class & $\begin{array}{c}\text { Redshift } \\
z\end{array}$ \\
\hline Mrk 421 & HBL & 0.030 \\
\hline Mrk 501 & HBL & 0.034 \\
\hline 1ES 2344+514 & HBL & 0.044 \\
\hline 1ES 1959+650 & HBL & 0.047 \\
\hline 1ES $1727+501$ & HBL & 0.055 \\
\hline BL Lacertae & LBL & 0.069 \\
\hline W Comae & IBL & 0.102 \\
\hline VER J0521+211 & IBL/HBL & 0.108 \\
\hline RGB J0710+591 & HBL & 0.125 \\
\hline H $1426+428$ & HBL & 0.129 \\
\hline B2 1215+303 & IBL/HBL & $0.130 ?$ \\
\hline 1ES 0806+524 & HBL & 0.138 \\
\hline 1ES $0229+200$ & HBL & 0.140 \\
\hline 1ES $1440+122$ & IBL/HBL & 0.162 \\
\hline RX J0648.7+1516 & HBL & 0.179 \\
\hline 1ES 1218+304 & HBL & 0.182 \\
\hline RBS 0413 & HBL & 0.190 \\
\hline 1ES 1011+496 & HBL & 0.212 \\
\hline 1ES 0414+009 & HBL & 0.287 \\
\hline 1ES $0502+675$ & HBL & $0.341 ?$ \\
\hline 1ES $0647+250$ & HBL & $\sim 0.45$ \\
\hline PG 1553+113 & HBL & $0.43<z<0.47$ \\
\hline $3 \mathrm{C} 66 \mathrm{~A}$ & IBL & $0.444 ?$ \\
\hline PKS 1424+240 & IBL/HBL & $>0.6035$ \\
\hline M87 & FR I & 0.0044 \\
\hline M82 & Starburst & 3.9 Mpc \\
\hline
\end{tabular}

Table 1: Extragalactic sources of TeV gamma-ray emission detected by VERITAS. Some redshifts are considered uncertain.

appear in stellar-mass black holes in X-ray binaries and GRBs. They are scaled-down versions of the jets seen in AGN. The observed similarity (in morphology and spectrum) of jets from black holes of different mass suggests that they share a common physical origin. VHE observations of AGN provide the opportunity to explore a large range of scientific topics, such as the study of the properties of SMBHs and their environments, indirect studies of the central engine and particle acceleration in relativistic jets. Open questions in AGN physics include the nature of particle acceleration and emission in jets, the structure and composition of jets, and with broad band blazar observations, we wish to constrain models of jet formation, acceleration, and collimation. Blazars are the best extragalactic probes of the extragalactic background light via their interaction with $\mathrm{TeV}$ photons traveling cosmological distances. Blazar observations have also been used to constrain the weak intergalactic magnetic fields (IGMF) (e.g. [6]), and test the validity of the Lorentz Invariance principle at high energies [7]. Blazars observations form a large part of the VERITAS program which includes a combination of planned blazar observations as part of a key science plan, long term monitoring of certain blazars, a discovery program for detecting new $\mathrm{TeV}$ blazars, and target-of-opportunity observations for flaring sources.

Table 1 lists all the extragalactic sources detected by VERITAS (as of July 2013). It includes a radio galaxy (M87), and a starburst galaxy (M82), but the majority are active galaxies of the blazar class. Of the 26 sources shown in the table, ten were discovered by VERITAS as TeV 
sources. A large fraction of the sources are high frequency peaked objects (HBLs), in which the peaks in the spectral energy distribution (SED) lie in the X-ray and TeV bands. In fact, the TeV blazar population is largely dominated by high-frequency peaked BL Lacs, but also includes low frequency peaked objects, flat spectrum radio quasars, and radio galaxies. In $\mathrm{HBL}$ objects, strongly correlated $\mathrm{TeV}$ and X-ray emission has been observed on many occasions, lending support to models in which the low-energy peak is explained by synchrotron emission from a population of ultra relativistic electrons, and the high-energy peak arises from inverse-Compton scattering of the synchrotron photons by the same population of electrons. Given better sensitivities than earlier imaging atmospheric Cherenkov telescopes, low synchrotron peaked blazars are becoming more common in $\mathrm{TeV}$ catalogs and several such sources are on the VERITAS blazar list. Typically, such blazars are detected mostly during flaring episodes/high flux states. Figure 4 shows a sky map of three blazars detected by VERITAS in the same field of view, illustrating the capability of VERITAS to detect faint gamma-ray sources at only a few percent of the Crab Nebula flux [8]. The three blazars shown in the figure are: W Comae, the first IBL detected at TeV energies [9], the HBL 1ES 1218+304 [10] and B2 1215+30 [11]. The blazars detected by VERITAS are predominantly nearby, with the range of red shifts from 0.03 to at least 0.60 with the upper limit coming from the recently discovered redshift of PKS 1424+240 (0.6035) [12]. In the following we describe five particular results from VERITAS. A more complete review of the VERITAS blazar program may be found in some recent publications ([13], [14]).

Radio Galaxy M 87: Among non-blazar extragalactic sources, the giant radio galaxy M 87 provides an unique opportunity to study VHE emission in close proximity to a super-massive black hole. M 87 is one of only four known VHE AGN with weak-to-moderate beaming; the other three are the radio galaxies Cen A, IC 310, and NGC 1275. The source has exhibited three distinct VHE flares with similarities in the VHE behaviour, but no unique multi-wavelength correlation. Figure 5a shows the multiwavelength light curves of M 87 over ten years of observations [15]. The exact location of the VHE gamma-rayemitting region in $\mathrm{M} 87$ remains elusive. $43 \mathrm{GHz}$ VLBA radio observations of the inner jet regions indicate no increased flux in 2010 [16], unlike the observations in 2008 where the VHE flare coincided with an enhancement of the radio flux of the innermost core regions. A flare in M87 in 2010 was jointly observed at VHE energies by VERITAS, MAGIC [17] and H.E.S.S. [18] and triggered an extensive multiwavelength campaign at lower energies. Figure $5 \mathrm{~b}$ shows results from the coordinated observation with VERITAS, H.E.S.S., and MAGIC during the bright VHE flare in 2010 [15]. This TeV flare was very well sampled, and the light curve shows a single isolated flare characterized by two-sided exponential decay $\tau_{\text {rise }} \sim 1.7$ days and $\tau_{\text {decay }} \sim 0.6$ days. Such day-scale variability is rarely seen in weakly-beamed sources.

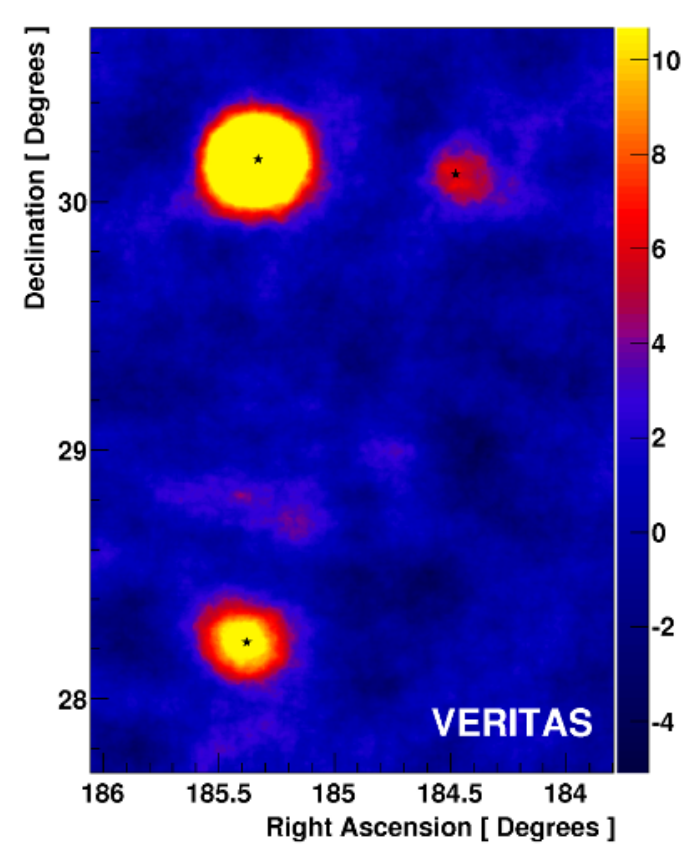

Figure 4. Significance map showing three blazars in a single VERITAS field of view. 1ES 1218+304 (upper left) is an HBL, while 1ES $1215+303$ (upper right) and W Comae are lowerfrequency- peaked BL Lacs. The color scale (shown on the right) gives the significance of detection. Note that the apparent extensions of the sources are due to saturation on the color scale; each blazar actually appears point-like at $\mathrm{TeV}$ energies. (Figure from [8]).

BL Lacertae: A recent highlight from VERITAS is the detection of the eponymous blazar BL Lac during a very bright, short-lived flare in 2011 June, the first detection of minute-scale variability in a low-frequency peaked BL Lac. The source is at a redshift of 0.069 and VHE emission from the source was discovered by MAGIC in 2005. VERITAS measured a flux of $\sim 125 \%$ of the Crab Nebula flux $(>200 \mathrm{GeV})$ during the flare, and a gamma-ray spectral index of $\Gamma=3.8 \pm 0.3$. Although the rising edge of the flare was not observed, during the short exposure of $\sim 35 \mathrm{~min}$, the flux was found to decay by factor of $\sim 3$ in $\tau=13 \pm 4 \mathrm{~min}$, placing strong constraints on the size of emission region (Figure 6a) [19]. Simultaneous changes in optical polarization, as well as the X-ray, optical and UV flux were observed. The VHE flare appeared during the same time as the emergence of a radio knot at $43 \mathrm{GHz}$ resolved as moving downstream in the jet, and the emergence of the radio knot was linked to the gamma-ray flare. Such correlated multiwavelength studies during a gammaray flare are important for locating the emission region in the blazar jet. Figure $6 \mathrm{~b}$ shows the spectral energy distribution (SED) of BL Lac both during and prior to the flare [19]. The TeV flare occurred when the source was active and variable in $\mathrm{GeV}$ gamma rays as detected by the FermiLAT. The simultaneous VERITAS and LAT [20] spectra show that the gamma-ray SED peak lies in the $\sim 10$ to 

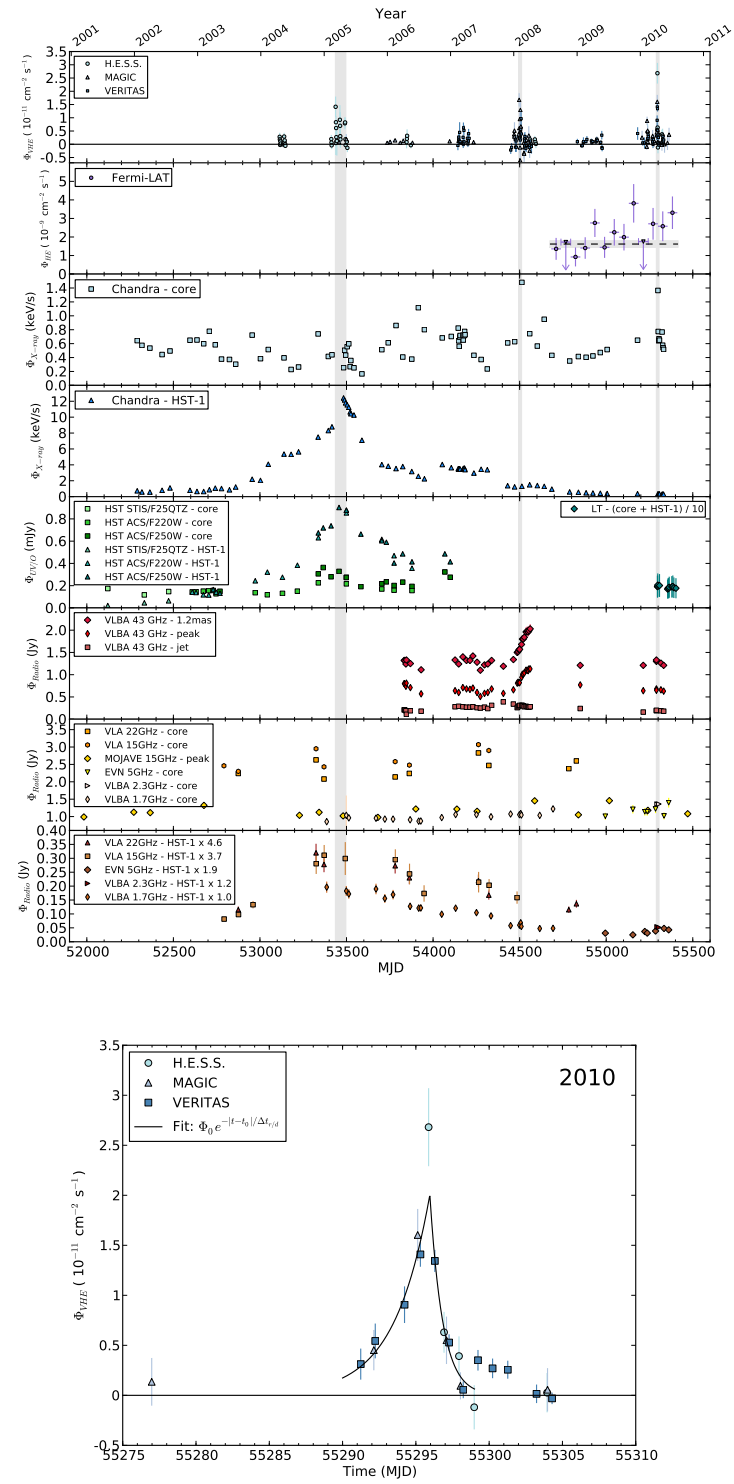

Figure 5. (a) (Top) Multiwavelength light curve of $M 87$ for a ten year period. The top panel shows the VHE gamma-ray flux calculated above an energy threshold of $350 \mathrm{GeV}$, as detected by VERITAS, MAGIC and H.E.S.S. For details on the data analysis and references see [15]. (b) (Bottom) A bright VHE flare in M 87 in 2010 recorded by VERITAS in a joint campaign with MAGIC and H.E.S.S., showing a variability timescale of $\sim 1$ day. The solid curve is the results of the fit of an exponential function to the data. (Both figures are from [15]).

$100 \mathrm{GeV}$ range. The LAT data show evidence for spectral hardening during the VERITAS flare.

VER J0521+211: The discovery of VER J0521+211 by VERITAS is an example of successfully finding new blazars behind the Galactic plane with the help of a FermiLAT trigger. A number of unidentified Fermi sources are expected to be blazars behind the Galactic plane. The characterization of blazars at low galactic latitudes is a difficult task because of optical extinction and the diffuse Galactic gamma-ray background. VHE telescopes such
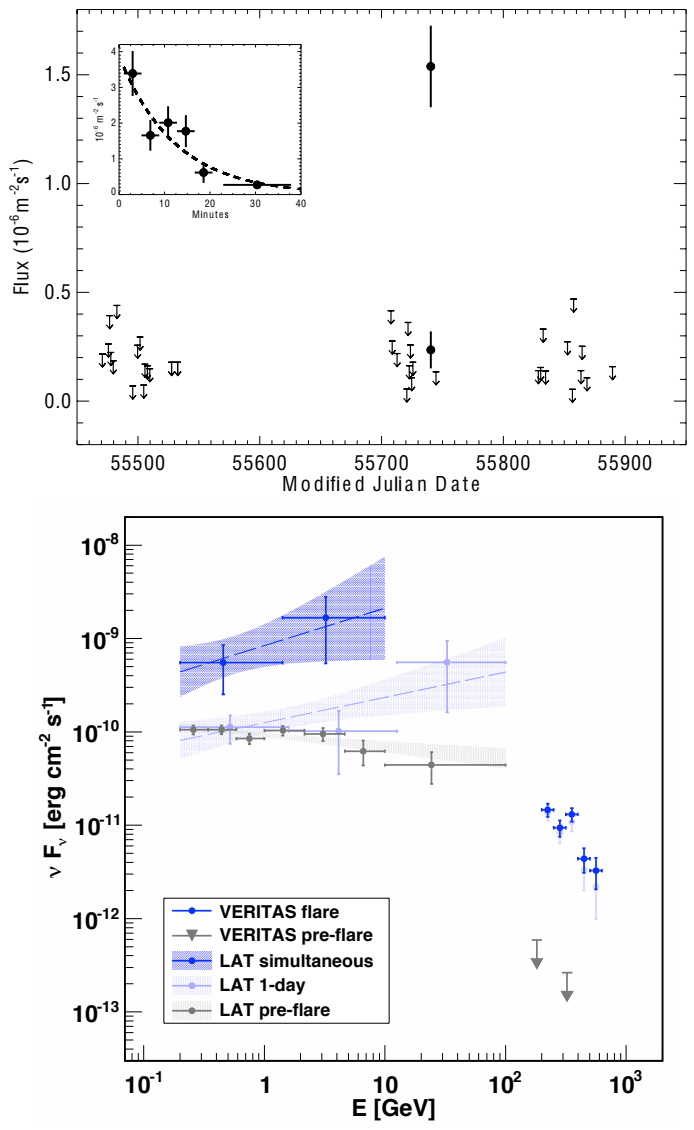

Figure 6. (a) (Top) VERITAS lightcurve of BL Lac in June 2011 $(>200 \mathrm{GeV}$ ). The inset shows the day scale variability in BL Lac. For details see [19]. (b) (Bottom) Broadband gamma-ray spectrum of BL Lacertae. For VERITAS, the flare spectrum with EBL correction is shown as blue points, with uncertainties, and the spectrum without EBL correction is shown as lighter blue points. The Fermi-LAT data shows hardening of the spectrum during the flare. (Both figures are taken from [19]).

as VERITAS are good tools for identifying new blazars at low latitudes with the help of better localization, and higher sensitivity to flux variability. Figure 7 a shows a map, in Galactic coordinates, showing the arrival directions of all diffuse class photon events with reconstructed energies greater than $30 \mathrm{GeV}$ recorded by Fermi-LAT in the first year of observations [21]. Observations of VER J0521+211 were motivated by the discovery of a cluster of $>30 \mathrm{GeV}$ photons in these data. VER J0521+211 was discovered at $\mathrm{TeV}$ energies in 2009 and was detected again in 2012 during a flare when its flux was $10 \%$ of the Crab Nebula flux. It was found to exhibit strong variability from optical to TeV bands, with a peak flux corresponding to $\sim 30 \%$ the steady Crab Nebula flux. Recent optical spectroscopy indicates that the object is a typical BL Lac with redshift $z=0.108$ [21]. Figure $7 b$ shows the spectral energy distribution of VER J0521+211 during the VERITAS detection in 2009 [21]. The high-energy emission from VER J0521+211 peaks in the gamma-ray band, between 10 and $200 \mathrm{GeV}$. Simultaneous flux mea- 

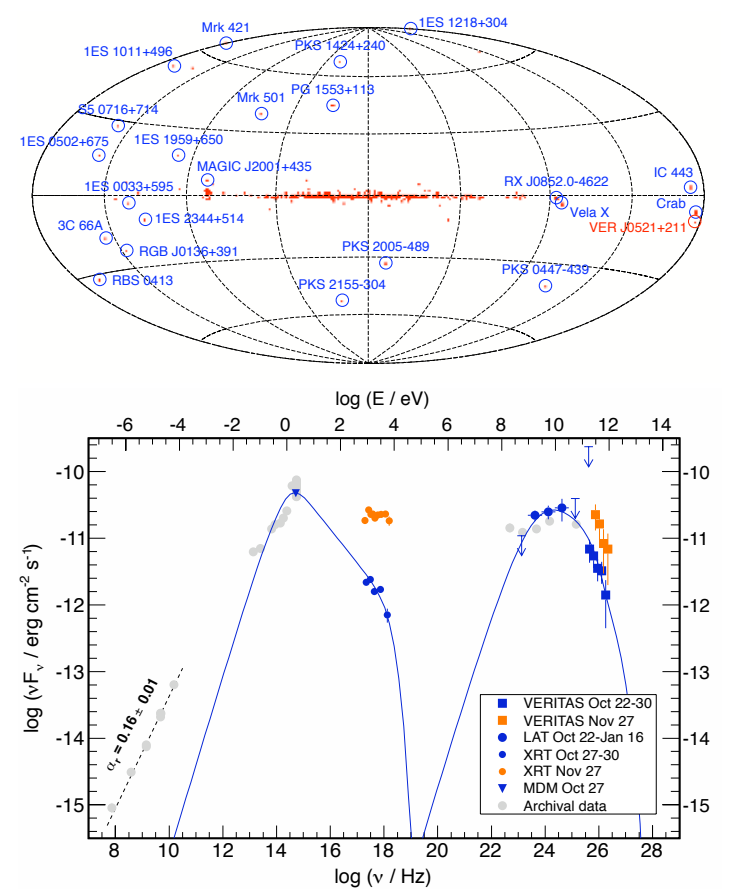

Figure 7. (a) (Top) Map in Galactic coordinates showing the arrival directions of all diffuse class photon events with zenith angle $<105^{\circ}$ recorded by Fermi-LAT in the first year of observations between 2008 Aug 4 and 2009 Aug 25, with energies greater than $30 \mathrm{GeV}$ [21]. (b) (Bottom) Broadband spectral energy distribution of VER J0521+211 during the VERITAS detection in 2009. The orange markers correspond to the flare data. The solid blue curve represents a one-zone SSC emission model with parameters adjusted to describe the low-state data. For details on the broadband data and modeling results, see [21]. Both figures are taken from [21].

surements during a "low" state (2009 October) suggest that VER J0521+211 has synchrotron properties similar to those of the known IBLs, although the synchrotron component shows HBL-like properties during the X-ray and $\mathrm{TeV}$ flare of 2009 November. A simple one-zone leptonic relativistic jet model which calculates the emission as synchrotron self-Compton radiation indicates a particledominated jet with a bulk Lorentz factor of 30, requiring a relatively weak magnetic field of $\sim 0.01 \mathrm{G}$.

Mrk 421: The well-known blazar Mrk 421 was the first VHE-detected extragalactic source and is typically the brightest blazar in the VHE band. The source is a subject of the VERITAS long-term blazar monitoring program and major flares were detected in 2008 and 2010 [22]. These initiated large multi-wavelength campaigns. Multiple outbursts have been observed to reach $\sim 10$ times Crab Nebula flux, allowing for studies with a high signal-tonoise ratio. In 2010 February VERITAS detected a strong, long-duration flare at $\sim 8$ Crab Nebula flux units when the gamma-ray flux was characterized in two-minute time bins. Recently in 2013 April, flaring was detected in the source during a multi-wavelength campaign with NuSTAR [23] and Swift [24], and the TeV flux was high over sev- eral nights, with strong intra-night variability. Analysis of this data set is underway.

1ES 0647+250 and 1ES 1011+496: Both these blazars were detected by VERITAS after the camera upgrade in 2012 and during observations carried out in partial moonlight [13]. VERITAS observations confirmed the prior discovery of these blazars as TeV sources by MAGIC. 1ES $0647+250$ was a promising VHE target based on its hard spectrum seen in the Fermi-LAT data. VERITAS observations yielded a detection at $2.9 \%$ of the Crab Nebula flux (> $140 \mathrm{GeV}$ ). In the case of 1ES 1011+496, VERITAS observed a flux $\sim 6.3 \%$ Crab Nebula flux above $150 \mathrm{GeV}$.

\section{Summary}

This article presents a brief summary of the VERITAS results on blazars from the past four years of observations. The population of VHE blazars detected by VERITAS is growing and VERITAS is expected to continue operations with the upgraded instrument for at least another five years with a strong focus on blazar observations. In addition, VERITAS has a coordinated effort to collect multi-wavelength data on blazars. Long term monitoring of a sample of northern VHE blazars will help in the study of spectral and flux variability. The observational goals of VERITAS are to catch fast flares (to study the size of the emission region), measure the SEDs of the brightest blazars and increase sensitivity to weak sources (to study emission mechanisms in blazars), and detect new soft-spectrum sources that may be accessible with the upgraded camera (to increase the number count and carry out blazar population studies). Studies of relativistic jets in blazars will remain a high priority for VERITAS.

This research is supported by grants from the U.S. Department of Energy Office of Science, the U.S. National Science Foundation and the Smithsonian Institution, by NSERC in Canada, by Science Foundation Ireland (SFI 10/RFP/AST2748) and by STFC in the U.K. We acknowledge the excellent work of the technical support staff at the Fred Lawrence Whipple Observatory and at the collaborating institutions in the construction and operation of the instrument.

\section{References}

[1] J. Holder, Proc. 32nd ICRC, Beijing, China (2011); arXiv:1111.1225.

[2] A. McCann et al., Astroparticle Physics, 32, 325 (2010).

[3] A. N. Otte, Proc. 32nd ICRC, Beijing, China (2011); arXiv: 1110.4702 .

[4] D. Kieda Proc. 33rd ICRC, Rio de Janeiro (2013); arXiv: 1308.4849.

[5] J. Holder et al., Astroparticle Physics, 25, 391 (2006).

[6] T. Arlen \& V. V. Vasiliev, 2012 Fermi Symposium proceedings, eConf C121028 (2012); arXiv:1303.2121.

[7] N. Otte et al., White paper submitted to Snowmass 2013, CF6 Cosmic Particles and Fundamental Physics (2013); arXiv:1305.0264.

[8] W. Benbow, Proc. 32nd ICRC, Beijing, China (2011); arXiv: 1110.0038.

[9] V. A. Acciari et al., ApJL, 684, 73 (2008). 
[10] V. A. Acciari et al., ApJL, 695, 1370 (2009).

[11] T. Arlen et al., ApJ submitted.

[12] A. Furniss et al., ApJL, 768, 31 (2013).

[13] J. Dumm, Proc. 33rd ICRC, Rio de Janeiro, Brazil (2013); arXiv:1308.0287.

[14] W. Benbow, Proc. 32nd ICRC, Beijing, China (2011); arXiv:1110.0038.

[15] A. Abramowski et al., ApJ, 746, 151 (2012).

[16] V. A. Acciari, et al., Science, 325, 444 (2009). Also, VLBA: http://www.vlba.nrao.edu/.
[17] MAGIC: https://magic.mpp.mpg.de/.

[18] H.E.S.S.: http://www.mpi-hd.mpg.de/hfm/HESS/.

[19] T. Arlen et al., ApJ, 762, 92 (2013).

[20] Fermi-LAT: http://www-glast.stanford.edu/.

[21] S. Archambault et al., ApJ, 776, 69 (2013).

[22] V. A. Acciari et al., ApJ, 738, 25 (2011).

[23] NuSTAR: http://www.nustar.caltech.edu/.

[24] Swift: http://swift.gsfc.nasa.gov/. 